\title{
Convincing Prospects to Switch Mobile Phone Providers: A Mind Genomics Cartography of an Everyday Opportunity to Optimize Messaging
}

\author{
Howard Moskowitz* \\ WICE, World Institute of Competitive Excellence, New York, USA \\ ${ }^{\star}$ Corresponding author: Howard Moskowitz, WICE, World Institute of Competitive Excellence, New York, USA
}

Received: October 19, 2021; Accepted: October 27, 2021; Published: November 03, 2021

\begin{abstract}
In a rapid, affordable, and scalable experiment, 50 respondents each evaluated unique sets of 24 'messages' dealing with 'offers to switch mobile phone providers'. The focus of the study was to show what could be learned from a simple experiment dealing with an everyday topic. For each test vignette of 2-4 messages, the respondents rated both likely to switch, and selected an emotion. The analysis revealed the emotional signature of each element, showing the feeling(s) most associated with each element, as well as the degree to which the element or message was rated as driving the respondent to switch providers. In terms of convincing respondents to say that they would be likely to switch providers, no elements performed strongly for the total panel. Only after clustering the respondents into four mind-sets by the patterns of their responses to the elements did the opportunities emerge, corresponding to specific messages. The paper shows the power and contribution of Mind Genomics to understanding a person's person's decision criteria, as well as providing immediate guidance to solve a practical problem. The attractiveness of the approach comes from the combination of the power of the approach, combined with the practical benefits of simplicity, speed, and affordability.
\end{abstract}

\section{Introduction}

Today (2021) it would be no exaggeration to say that the smart phone is ubiquitous. The millennial generation has grown up with the smart phone. It is unimaginable to many that there could have been an era when the telephone was just that, a device into which people spoke to other people either on the next street, or more rarely in the next state, or even more remarkably (and with great expense) people living in a foreign country. Those who are old enough to marvel at the change in technology like to compare the technology of today's smart phone with the available technology in 1969, when John Glenn went to the moon and back.

As a consequence of the accelerated acceptance of the smart phone, the phone itself has become a commodity. What attracted attention 30 years ago in the 1990's is now a part of everyday life? And of course, with the ubiquity of the smartphone is the ubiquity of the provider. The smartphone is everyone's entry point into today's powerful technology. Virtually world-wide people can do things such as make video calls at low price, and with the abandon of something which is virtually free.

A search through today's literature (Google Scholar ${ }^{\oplus}$, done October 18, 20201) for the topic of 'switching mobile phone providers' showed an astonishing 57,800 hits, and changing the word from provider to carrier reduced the number of hits to 38,000 . The numbers become even more remarkable when we break up to 'hits' for mobile phone providers, doing the analysis in by five-year periods, and then compute the approximate hits/month. Table 1 shows these statistics.
Table 1: Hits for 'switching mobile phone providers'.

\begin{tabular}{|l|c|c|}
\hline Period & Total Hits & Hits/Month \\
\hline $1990-1994$ & 634 & 11 \\
\hline $1995-1999$ & 2450 & 41 \\
\hline $2000-2004$ & 6700 & 112 \\
\hline $2005-2009$ & 10,900 & 182 \\
\hline $2010-2014$ & 15,900 & 265 \\
\hline $2015-2019$ & 16,700 & 278 \\
\hline $2020-2021$ & 7,390 & 308 \\
\hline
\end{tabular}

Clearly the academic literature reflects the interest in mobile telephony, and the services provided. What is just as interesting are the topics. What emerge from the literature search is what might be expected, namely studies of the topic in different countries, namely what are the important aspects to which people attend. Here are two representative titles of papers. The focus is on the general process of how people think about the issue of switching:

Switching behavior of mobile users: do users' relational investments and demographics matter? [1]

Drivers of brand switching behavior in mobile telecommunications. [2]

The search through many of the Google Scholar ${ }^{\oplus}$ hits reveals two patterns. The first pattern is the academic effort to simplify the topic of switching into a set of actions, or need states, viz., the need to 
systematize understanding. The second pattern, far more frequent, is the analysis of switching behavior, along with motivations for doing so, in the many countries around the world. This second effort gives the reader an awed sense of the power, and the ubiquity of mobile telephony.

What is missing, however, is the practical understanding of the type of messaging which drives consumers to feel that they would switch mobile phone providers. The literature may contain some of these messages, but the focus is generally from the 'outside in', viz., looking at the patterns of behavior in the increasingly important world of mobile telephony. Like so many other areas, the focus ends up rarifying the specifics, the messages, which become ephemera, to be discarded in the search for lasting 'truth' or at least 'general patterns.'

The focus on Mind Genomics is on these ephemera, specifically the messaging. The objective of Mind-Genomics, as emphasized below, is the understanding of the messaging, and by so doing, understanding the topic from the 'inside-out', from the mind of the customer faced with the array of messages, and the competing cacophonies of merchants hawking their wares, shouting their offers.

Faced with this topic, the experiment reported here, done 10 years ago, is still relevant. The technology may have changed, but as we will see, the minds of people then made sense. Nothing discovered a decade ago has not changed very much, nor surprises very much. In this paper we resurrect data a decade old to show how understanding the way the consumer mind works provides data which has a very long shelf-life. Underneath the technology is the benefit, the appreciation of that which does not change from year to year.

\section{Ways to Solve the Problem}

Better messaging to convince buyers is a hallmark topic of consumer research. The publications one sees in the scientific and business literature about the topic is dwarfed by the amount of information retained in the archives warehousing corporate research. It should come as no surprise that the proper messaging of a company's offer is a key to attracting customers, at least new customers, who have almost no other opportunity to know what is available unless they are told.

The above being said, it is rare that a corporate executive will truly know the messages which appeal to customers. There may be some answers of an obvious nature, but the reality is that most of the corporate knowledge is based upon such things as 'this is the way we have advertised before.... it works. ...let's not take a chance, let's not change.' Of course there are situations such as the recent Covid-19 pandemic which has changed the way people behave, but there is the seemingly eternal reticence to explore new ideas.

The typical approach to testing messages, so-called 'promise testing', evaluates the messages one at a time, looking for messages which either simply score well, or upon probing, appear to convey messages of the right tonality. It is often averred by the advertising agency and by marketing gurus that one requires a sensitive and developed ear and mind to 'know' what will succeed in the marketplace, and that simply testing many messages does not reveal the truly breakthrough idea. The result of such statements is the continuing fight between the artist who 'knows' what will work, and the researcher, who must test to know what will work. The artist feels that one or two of the 'right talented' individuals, like the copyrighter, can do the job. The researcher feels that it will require a representative group of target consumers to ensure that one is making the correct choice.

\section{The Mind Genomics Process}

The Mind Genomics process has evolved over the past thirty years, during which time is has evolved into the beginning of a science, with many published paper, and an increasing cadre of practitioners around the world [3-6].

The Mind Genomics process is a systematized, templated process, requiring the researcher to break up the problem into a series of small, easily managed steps. By so doing, Mind Genomics forces the researcher (really the user) to think in both a creative and a structure way, respectively. The steps below, moving from thinking to discovery, were done over a period of two days. Today, a decade later, the process would be reduced to about two hours.

\section{Step 1 - Create Materials}

Table 2 shows the set of four questions and six answers for each question. Mind Genomics forces the researcher to think a structured fashion, beginning with a topic, continuing with a set of questions, and then for each question a set of alternative answers. The Mind Genomics system comprises a variety of different experimental designs, layouts or sets of combinations comprising a specific number of 'questions' and for each question a set required number of 'answers'. The array shown in Table 2 is called a 4x6 (four questions, each with six answers). Today's practice has been reduced to the much easier and faster design, the $4 \times 4$ (four questions, each with four answers).

The questions in Table 2 'tell a story'. There is no need for the researcher to deeply understand the topic in order to develop the questions and the answers to the questions. Rather than forcing the researcher to select the answers that will be best, thus delaying the process until everything is 'just right', the Mind Genomics process has been designed to be simple, affordable, and iterative. The researcher is encouraged to 'just do it', find the results, and 'do it again, changing what didn't work with new guesses. Thus, Table 1 presents a 'first guess.' With 'n' cycle time of 1-2 hours, the second iteration would see some new questions and answers replacing the ones which performed poorly, viz., simply 'did not convince the respondents'.

As one might surmise, the hardest part of this first section is coming up with the four questions which 'tell a story'. The questions require the researcher to think more deeply and critically about the topic. When presented with the notion about asking a question, most researchers begin with a question that can be answered yes or no, or with some specific one-word answer. It takes a while for the researcher to think in terms of questions which require a phrase as an answer, rather than a simple no/yes. In contrast, once the questions are asked, the answers in the form of a declarative phrase are easy to create. The questions provide the structure for the answer. 
Howard Moskowitz (2021) Convincing Prospects to Switch Mobile Phone Providers: A Mind Genomics Cartography of an Everyday Opportunity to Optimize Messaging

Table '2: The four questions and the six answers for each question. Brand names are disguised.

\begin{tabular}{|c|c|}
\hline & Question A: How do we allay your concerns about our support? \\
\hline Al & Hundreds of technical support staff only a phone call away \\
\hline A2 & Check service problems online! Our online support staff can keep you updated on all service problems \\
\hline A3 & Connect with fellow network members via our online forums \\
\hline A4 & Stores everywhere to help you find the right phone \\
\hline A5 & Check your phone and voice your concerns at any of our retail stores \\
\hline \multirow[t]{2}{*}{ A6 } & Reasonably priced extended warranties for all of our phones \\
\hline & Question B: What are the 'fun' features of our phones? \\
\hline B1 & Most of our phones come with pre-installed cameras, games, and applications. \\
\hline B2 & Buying applications for phones is simple, easy, and inexpensive \\
\hline B3 & Numerous applications ranging from calculators to puzzles and games. \\
\hline B4 & All compatibility questions can be easily answered online on our website. \\
\hline B5 & Applications can be purchased online and downloaded immediately to your phone. \\
\hline \multirow[t]{2}{*}{ B6 } & Many different pricing options for applications -from monthly subscription to one-time fees . \\
\hline & Question C: What prices do we feature? \\
\hline $\mathrm{Cl}$ & Individual plans start as low as $\$ 39.99$ a month \\
\hline $\mathrm{C} 2$ & Family plans starting from $\$ 59.99$ \\
\hline $\mathrm{C} 3$ & Don't want a plan... pre-paid service for only $\$ 2$ a day \\
\hline $\mathrm{C} 4$ & A 2-gigabyte data plan for only $\$ 35$ a month \\
\hline C5 & Inexpensive plan for international calls \\
\hline \multirow[t]{2}{*}{ C6 } & Mobile Broadband Plan enables you to use internet on your (Product 1) or any other smartphone \\
\hline & Question 4: What are some other fun features? \\
\hline D1 & Various phones featuring slide out keyboards \\
\hline D2 & Don't like too many buttons We also carry simple touch tone phones \\
\hline D3 & Buy one of our phones and immediately begin texting \\
\hline D4 & Star Wars lover... Our new (Product 2) has an awesome Star Wars theme \\
\hline D5 & Choose from our wide (Product 3 ) selection for web browsing and mobile apps \\
\hline D6 & Our amazing phone also features a slide out keyboard \\
\hline
\end{tabular}

\section{Step 2 - Creating an Experimental Design Which Mixes and Matches 2-4 Elements in Each Element}

The hallmark of 'field work' in Mind Genomics is the evaluation of combinations of elements (so-called vignettes), with these vignettes systematically composed according to an underlying experimental design [7]. The design specifies the composition of each vignette. No effort is made to link together the elements of the vignette, an example of which appears in Figure 1.

Each vignette comprises a specified number of 2-4 elements. Each respondent evaluates 48 vignettes. The mathematical structure of the 48 vignettes is the same from one respondent to another with the mathematical structure ensuring that the 24 elements are statistically independent of each other. The Mind Genomics system presents each respondent with a totally new set of combinations of the 24 elements but a set of combination following the SAME underlying structure. Although the structure remains the same, the actual combinations different. Rarely do respondents ever test the same combinations. That difference in combinations is a property of the underlying design [8].

\section{Step 3 - Execute the Study, through a Third Part On-line Field Service}

Since the early years of this century, on-line panel providers have made available respondents to participate in studies conducted on the internet. What was unusual in the late 1990's is today the 'norm.' Most people have been invited to participate in a variety of 'studies', whether these studies deal with limited topics such as the satisfaction of their last transaction with a company, or the studies deal with longer, more involved topics conducted as polls. In contrast, the Mind-Genomics process can be thought of as an experiment conducted on the internet, but in the form of a simple set of answers to systematically varied stimuli.

The respondents received an invitation to participate in the study, which would last about 10-15 minutes. The respondents interested in the study pressed on the linked embedded in the email invitation, and were taken to the study. The study began with an orientation, which is shown in Figure 1. The orientation screen used at the time of the study in 2012 was substantially longer than the orientation screen 


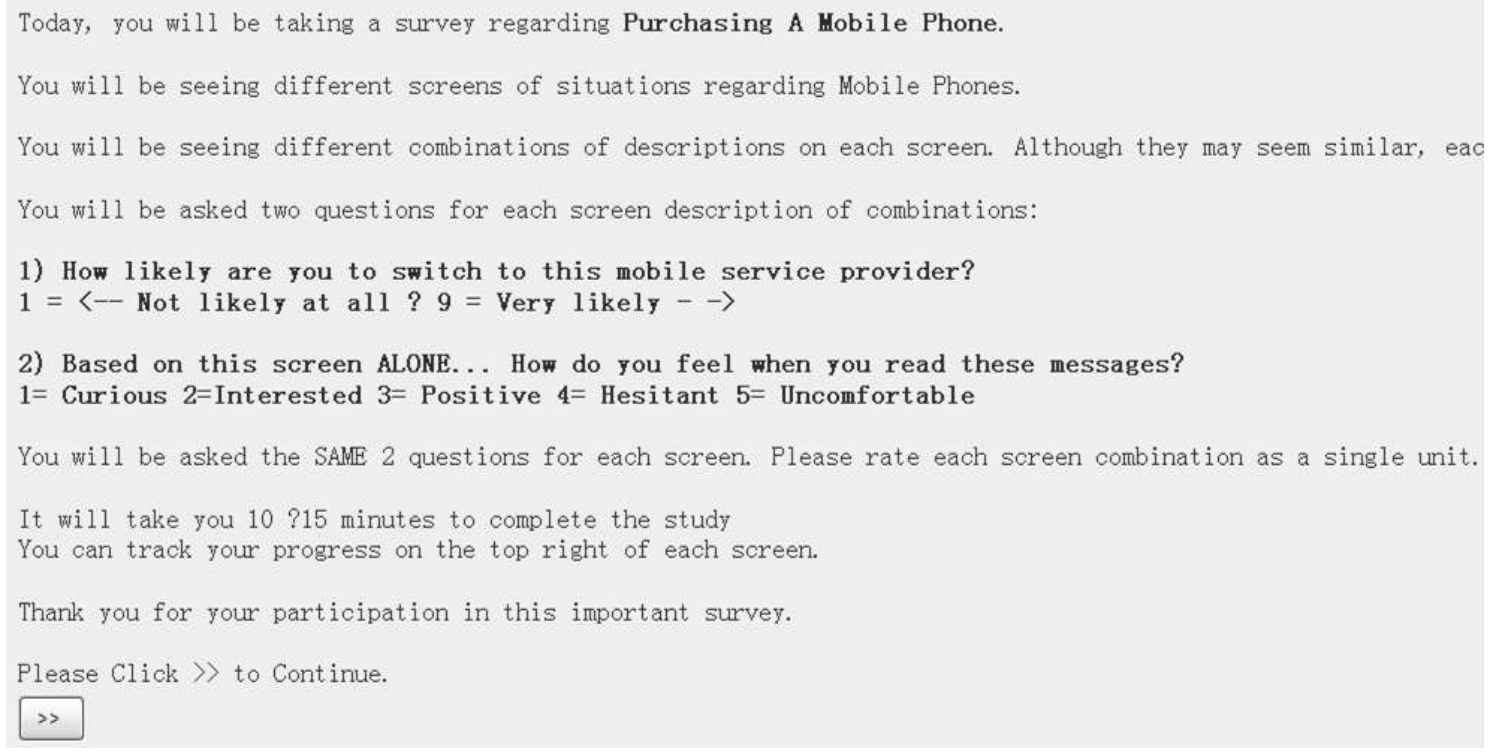

Figure 1: The orientation page to the Mind Genomics study on purchasing a mobile phone.

used today. Of special interest is the effort to reassure respondents that they are evaluating different vignettes. This effort to communicate that all the vignettes are really different from each came from a few complaints from professional and students that the vignettes seemed all the same. The early efforts in Mind Genomics focused on establishing the usefulness of the approach, one way of doing so being an effort to anticipate problems and avoid them. Thus the effort to reassure the respondent that the vignettes all differ from each other. Another remnant of the early effort, still in force, is the reassurance that the evaluations will last 10-15 minutes. This effort comes from the complaint from some professionals (but not panel respondents) that the effort is 'overly long', and that 'how much is left?'

The IdeaMap program presented the respondent with a set of 48 vignettes, each vignette to be rated on two scales. The first scale instructed the respondent to rate the vignette on likelihood to switch to the provider. The second scale instructed the respondent to select one of five emotions experienced after reading the vignette.

Figure 2 shows an example of the vignette, set up for Rating Scale \#1 (how likely are you to switch to this mobile service provider?). The combinations of elements are dictated by the specific permuted design for the respondent. The program makes no effort to beautify the combination, viz., by providing connections between the elements. The elements are presented as centered phrases, in unadorned fashion. Despite the apparent starkness of the stimulus, few respondents complain. Rather, over the $40+$ years that this format has been used (since 1980), many respondents have made the unsolicited comment that the format actually helped them to 'scan' the vignette, and make their decision.

For each vignette, the respondent assigned two ratings, for the likelihood of switching, and then for the selection of emotion. The vignette remained the same. As soon as the respondent rated the vignette by selection the closest emotion to what was being experienced, the vignette closed, and the next vignette was immediately presented. At the end of the evaluation, the respondents provided answers to four classification questions, including gender, age, and two on patterns of usage.

\section{From Vignette to Mind - The Templated Analyses of Mind Genomics}

The focus of Mind Genomics moves to the elements. The vignettes are only a convenient way to ensure that the elements are presented in a more typical fashion, approximating the typical type of offer, rather than being presented one-at-a-time. Presenting vignettes, all differing from each other, makes it almost impossible for the respondent to be politically correct, to 'game the system,' and provide the answers that one might deem to be 'the right answer.'

Given focus on individual elements, Mind Genomics moves from the combinations, the vignettes, to deconstructing the vignettes into the contributions of the individual elements. Recall that each respondent evaluated 48 different combinations, and that the elements appeared 2-4 times in the combinations. Furthermore, each element appeared an equal number of times, and the elements appeared in an uncorrelated fashion.

The analysis begins by creating a data matrix, each row corresponding to one vignette from one respondent. The matrix comprised a column to identify the respondent, 24 columns corresponding to the 24 element, and two final columns corresponding to the rating assigned on Rating Scale \#1 and Rating Scale \#2, respectively. The final four columns contained the answers to the selfprofiling questions (age, gender, and two questions about phone use).

The data matrix comprising 1's (element present in vignette) and 0's (element absent from vignette) presents the 24 elements as so-called dummy variables, absent or present. There is no metric information about the dummy variables. The objective of the analysis is to determine the degree to which the element drives estimated switching (Rating Scale \#1) or links with certain feelings/emotions (Rating Scale \#2). 


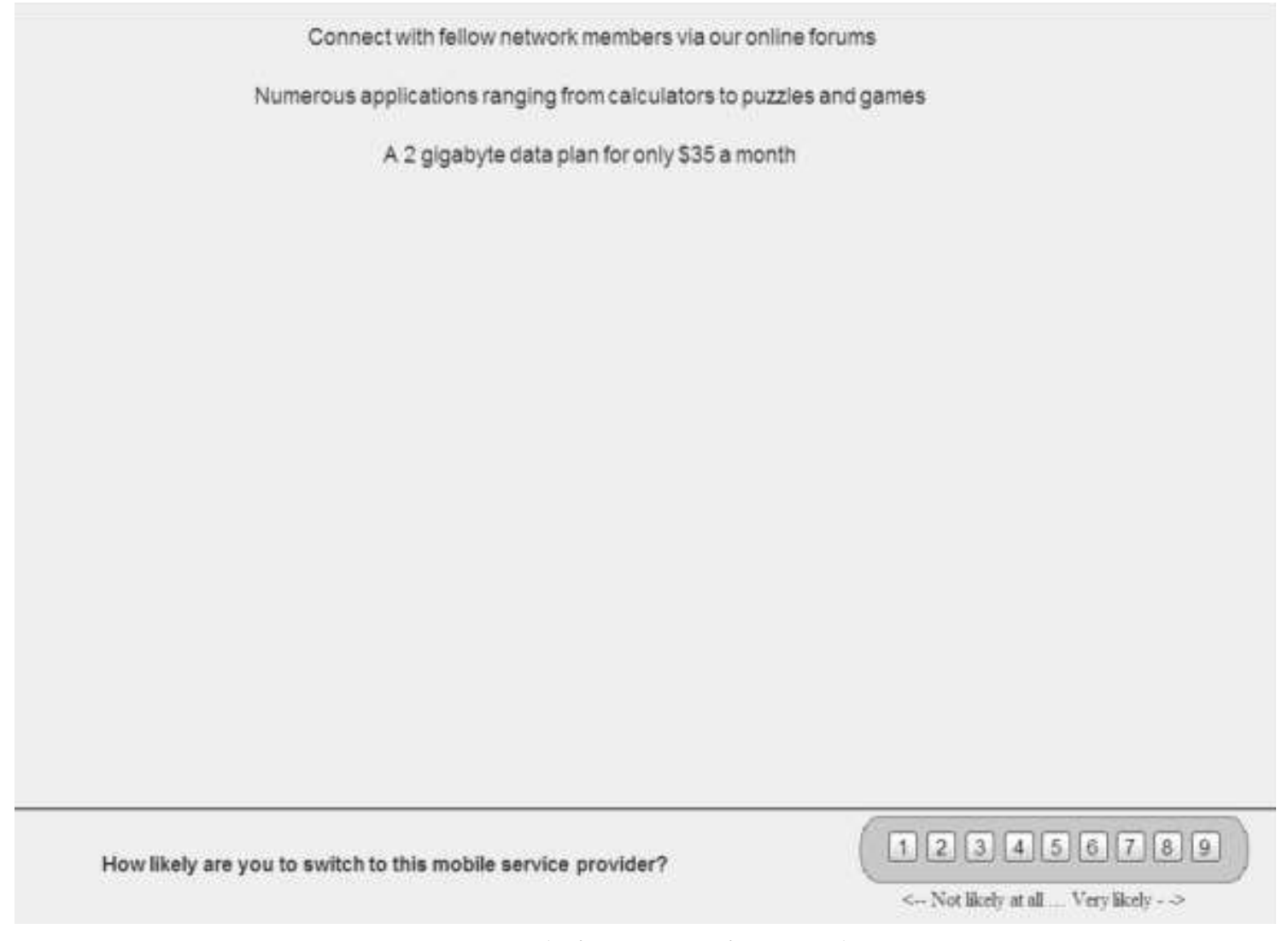

Figure 2: Example of a vignette set up for Rating Scale \#1.

The actual data matrix comprises a set of 48 rows for each of the 50 respondents, or 2400 rows of data.

The 9-point rating for Rating Scale \#1 (1=not likely to switch at all ... 9=very likely) is converted to a binary scale, with ratings of 1-6 converted to 0 , and ratings of 7-9 converted to 100 . To each of the newly created binary numbers is added a very small random number $\left(<10^{-5}\right)$.

The rationale for converting the 9-point scale to a binary scale is the proclivity of users of data to demand simple yes/no statements, viz., will the respondent switch or not switch, based upon the elements in the vignette? It is technically correct to say, 'the data shows a rating of 7, closer to switch and further away from not likely to switch. That answer is not useful in a business situation, where the answer should be all or none. The 9-point Likert scale could be replaced by a simple binary scale (no/yes) at the outset, but there is always interest in precision for other analyses that may be of interest.

The same type of transformation is done for the emotions, except that five new binary variables are created, one each for curious, interested, positive, hesitant, and uncomfortable, respectively. For each vignette, the emotion selected in Rating Scale \#2 is given the value 100, and the four emotions not selected in Rating Scale \#2 are each given the value 0. Again, and afterwards, each of the five newly created emotion variables has another vanishingly small random number added.

The rationale for adding a small random number to each newly created binary variable is ensure that the binary variables exhibits some small degree of variation each at the individual level. Were a single respondent to rate all the vignettes 1-6, for instance, or select the emotion 'hesitant', the conversion would transform all of the respondent's ratings into the same value. The OLS (ordinary leastsquares) regression would fail. Adding a the vanishingly small random number is a prophylactic step, not affecting the results, but protecting against a crash of the regression program used to relate the elements to the ratings.

\section{Linking Emotions to Elements}

Our first analysis focuses on the link, if any, between the element and the selection of an emotion. Recall that the respondent selected one feeling/emotion for each vignette. The analysis is straightforward, promoted by the foresight of creating the vignettes according to the permuted experimental design.

The analysis creates five OLS (ordinary least squares) regression equations, each expressed in the same way: Linkage (to an emotion) = $\mathrm{k}_{1}(\mathrm{~A} 1)+\mathrm{k}_{2}(\mathrm{~A} 2) \ldots \mathrm{k}_{24}(\mathrm{D} 6)$

The foregoing equation says that the linkage between the feeling/ emotion and be expressed by a simple equation, which shows the linkage of each element to the emotion. Coefficients $\left(\mathrm{k}_{1}\right.$ to $\left.\mathrm{k}_{24}\right)$ are estimated using OLS regression, without estimating the additive constant. Coefficients of about 10 or higher are statistically significant and relevant, based upon previous observations across many projects using Mind Genomics conjoined with ratings of emotion. 
Howard Moskowitz (2021) Convincing Prospects to Switch Mobile Phone Providers: A Mind Genomics Cartography of an Everyday Opportunity to Optimize Messaging

For our presentation here, and to allow the strong patterns to emerge, we show the data from the total panel, but show only those coefficients or linkages 10 or higher. Table 3 shows those strong linkages. The elements not appearing at all in the table are those which do not show a strong linkage to the feeling/emotion. nt.
The important thing to note is that improvement in our understanding of the elements, simply by learning the linkage of emotions and elements. From the entire array of $50 \times 48$ or 2400 vignettes we see that despite the imagined difficulty of the task, the linkages exhibit face validity, making sense.

Table 3: Linkage between elements and feelings/emotions. Only strong linkages of 10 or are shown.

\begin{tabular}{|c|c|c|}
\hline & Curious & \\
\hline \multirow[t]{2}{*}{$\mathrm{C} 4$} & A 2-gigabyte data plan for only $\$ 35$ a month & 10 \\
\hline & Interested & \\
\hline C4 & A 2-gigabyte data plan for only $\$ 35$ a month & 13 \\
\hline \multirow[t]{2}{*}{$\mathrm{C} 1$} & Individual plans start as low as $\$ 39.99$ a month & 10 \\
\hline & Positive & \\
\hline A2 & Check service problems online! Our online support staff can keep you updated on all service problems & 16 \\
\hline A4 & Stores everywhere to help you find the right phone & 14 \\
\hline D6 & Our amazing phone also features a slide out keyboard & 14 \\
\hline Al & Hundreds of technical support staff only a phone call away & 12 \\
\hline A3 & Connect with fellow network members via our online forums & 12 \\
\hline A5 & Check your phone and voice your concerns at any of our retail stores & 12 \\
\hline B3 & Numerous applications ranging from calculators to puzzles and games. & 12 \\
\hline C6 & Mobile Broadband Plan enables you to use internet on your (Product 1) or any other smartphone & 11 \\
\hline D1 & Various phones featuring slide out keyboards & 11 \\
\hline $\mathrm{C} 1$ & Individual plans start as low as $\$ 39.99$ a month & 11 \\
\hline B1 & Most of our phones come with pre-installed cameras, games, and applications . & 11 \\
\hline \multirow[t]{2}{*}{ A6 } & Reasonably priced extended warranties for all of our phones & 10 \\
\hline & Hesitant & \\
\hline A6 & Reasonably priced extended warranties for all of our phones & 18 \\
\hline B5 & Applications can be purchased online and downloaded immediately to your phone : & 16 \\
\hline D3 & Buy one of our phones and immediately begin texting & 16 \\
\hline D4 & Star Wars lover... Our new (Product 2) has an awesome Star Wars theme & 15 \\
\hline $\mathrm{C} 2$ & Family plans starting from $\$ 59.99$ & 15 \\
\hline C3 & Don't want a plan... pre-paid service for only $\$ 2$ a day & 15 \\
\hline D6 & Our amazing phone also features a slide out keyboard & 15 \\
\hline D2 & Don't like too many buttons We also carry simple touch tone phones & 14 \\
\hline C5 & Inexpensive plan for international calls & 14 \\
\hline B4 & All compatibility questions can be easily answered online on our website. & 14 \\
\hline A4 & Stores everywhere to help you find the right phone & 13 \\
\hline A3 & Connect with fellow network members via our online forums & 12 \\
\hline D5 & Choose from our wide (Product 3 ) selection for web browsing and mobile apps & 11 \\
\hline B2 & Buying applications for phones is simple, easy, and inexpensive & 11 \\
\hline B6 & Many different pricing options for applications -from monthly subscription to one-time fees . & 11 \\
\hline A2 & Check service problems online! Our online support staff can keep you updated on all service problems & 10 \\
\hline A1 & Hundreds of technical support staff only a phone call away & 10 \\
\hline B3 & Numerous applications ranging from calculators to puzzles and games & 10 \\
\hline \multirow[t]{2}{*}{$\mathrm{C} 1$} & Individual plans start as low as $\$ 39.99$ a month & 10 \\
\hline & Uncomfortable & \\
\hline D4 & Star Wars lover... Our new (Product 2) has an awesome Star Wars theme & 10 \\
\hline
\end{tabular}


The elements which are interesting are pricing.

The elements which are strongly positive are first service, and then features.

Sometimes an element links to a positive element and to a slightly negative element (hesitant). For some people the element (Reasonably priced extended warranties for all of our phones) provokes the feeling of interested, for others the same element provokes the feeling of hesitant. There may be different mind-sets among the respondents, viz., and ways of thinking.

Finally, one message actually makes the respondent feel uncomfortable (Star Wars lover... Ournew (Product 2) has an awesome Star Wars theme)

\section{Linking the 24 Elements to the Likelihood of Switching}

We now return to the original focus of the study, viz.., what messages, if any, are likely to get a person to consider switching mobile phone providers. Although the study focused on mobile phone providers, the question is universal in the world of business. The Mind Genomics process provides an approach to answer the question, doing so quantitatively and efficiently.

The analysis once again begins with a transformation, this time with ratings of 1-6 transformed to 0 , rating 7-9 transformed to 100 , and a vanishingly small random number added to each of the transformed ratings. There is not fixed about the criteria of transformation, but the bifurcation of 1-6 and 7-9 has been the standard one for decades. Sometimes the division is at 7 (1-7 transformed to $0 ; 8-9$ transformed to 100). This is done for respondent populations which tend to up-rate vignettes, and corrects for the exceptionally large number of positive responses.

The analysis creates one OLS regression equation, of the form: Likely to switch $=\mathrm{k} 0+\mathrm{k}_{1}(\mathrm{~A} 1)+\mathrm{k}_{2}(\mathrm{~A} 2) \ldots \mathrm{k}_{24}(\mathrm{D} 6)$

This time the equation has an additive constant. The additive constant, $\mathrm{k}_{0}$, is a measure of the likelihood to switch in the absence of elements. Of course, by design all the vignettes comprised 2-4 elements, so there are no vignettes without elements. Nonetheless, the additive constant gives a good sense of the likely reception of one's offers, information valuable to have in a marketing campaign. Without a Mind Genomics experiment of this sort, one would have to ask a respondent directly, or mine the switching data of the respondent. With Mind Genomics the additive constant convenient provides this measure of proclivity to switch. The above-mentioned equation is calculated at the level of the group, with the group defined by total, by specific ages, and by gender, respectively.

When the topic is switching, the information emerging from the analysis suggests the following findings, information that would be useful both to the marketer facing the business problem, but also to the researcher trying to understand what motivates people. Table 4 shows the relevant elements of the model for five groups; total, two genders, two age groups, respectively. There are only 42 of the 50 respondents shown in the age groups. The remaining groups, younger and older, did not comprise a sufficient number of respondents to show.

We begin with the additive constant. As noted above, the additive constant is the estimates likelihood of switching providers in the absence of messages, and should be considered a baseline. Table 4 shows a basically low likelihood of switching in the absence of a compelling message, with the additive constant of 20 for the total panel. Females are more likely to switch than males are (constant 29 vs 21). The age groups are similar, although the older respondents are slightly less likely to switch.

At this point, the common criticism is the small base size. With larger base sizes the additive constant will remain the same, with the usual 'variability' encountered with subjective data. What is important is that a 3-4 hours excursion into an experiment suggests topics, messages, and even opporgtunities, perhaps even not hitherto expected or perhaps conjectured but not demonstrated.

The body of Table 4 shows only those elements which generate a coefficient of +6 or higher for any one of the five groups. Only four elements do so, with the male respondents being most positive. For the total panel and for females no elements generate strong performing coefficients.

The low additive constant and the lack of strong performing elements among the total panel and key demographic subgroups are not the results of a low base size. That is, increasing the number of respondents from 50 to 100 or even 200 or 500 is not likely to produce results too different from what we see in Table 4. We conclude, therefore, that there are simply no elements which really drive switching, and that the team must go back to create new messaging. It is better to find this information out in an hour or two than in a month or two.

Table 4: Additive constant and strong performing elements for total panel, gender, and age. Only elements showing a coefficient of +6 or higher are shown.

\begin{tabular}{|c|c|c|c|c|c|c|}
\hline & & & \multicolumn{2}{|c|}{ Gender } & \multicolumn{2}{|c|}{ Relevant Ages } \\
\hline & & Total & Male & Female & $26-40$ & 41-55 \\
\hline & Base Size & 50 & 21 & 29 & 23 & 19 \\
\hline & Additive constant (basic likelihood of switching) & 20 & 23 & 18 & 23 & 13 \\
\hline A1 & Hundreds of technical support staff only a phone call away & & 8 & & & \\
\hline A2 & Check service problems online! Our online support staff can keep you updated on all service problems. & & 7 & & & \\
\hline $\mathrm{C} 1$ & Individual plans start as low as $\$ 39.99$ a month & & 13 & & 8 & \\
\hline $\mathrm{C} 4$ & A 2-gigabyte data plan for only $\$ 35$ a month & & 12 & & & 6 \\
\hline
\end{tabular}


Howard Moskowitz (2021) Convincing Prospects to Switch Mobile Phone Providers: A Mind Genomics Cartography of an Everyday Opportunity to Optimize Messaging

There is, however, another possibility, viz., that there are different ways of thinking about the offers, ways which do not emerge when we just know age and gender. This is known as mind-sets,

Clustering uncovers hitherto unexpected groups of people in the population, mind-sets in the language of Mind Genomics. Within a cluster the respondents see the world similarly, at least the world of offers regarding switching mobile phone providers. Clustering does not pretend that these mind-sets are actually fixed in stone. Rather, clustering is an analytic 'heuristic', trying to make sense out of variation which inevitable occurs in data concerning choices. The clustering provides insights which would otherwise not emerge

The mechanics of clustering is straightforward. There are different ways to cluster data, all of which are equally 'correct,' but simply a matter of decision. Clustering attempts to uncover groups in the data, not based on who the groups ARE but rather on how the groups perform.

The mechanics of clustering begins with the generation of the information on which the clustering will be done, such information obtained at the level of the individual respondent. The subsequent analysis creates the clusters or mind-sets. Recall that each respondent tested the same structure of 48 combination, prescribed according to by a single experimental design that was permuted to create new combinations evaluated by each respondent. . The design allows for the estimation of individual-level models or equations, just as we estimated the group model. Thus, the foregoing equation with the additive constant is created at a respondent-by-respondent level to provide a matrix of 50 rows, one per respondent, and 25 numbers in each row, the additive constant and the 24 coefficients. That data matrix is then analyzed by through clustering, using the 24 coefficients (but not the additive constant) to define first two clusters, then three clusters, then four clusters of respondents. A cluster comprises individuals whose patterns of coefficients are similar to each other, and quite dissimilar to the coefficients of the respondents in the other clusters [9].

Table 5 shows new opportunities for messaging when we break the respondents into mind-sets based upon the pattern of responses to the messages that a mobile provider would likely use With 50 people the objective is not to create the science of messaging for this topic, but rather at a tactical level to identify messages which seem to work. There are certainly no promising messages when we look at the Total Panel. When we move to two mind-sets we three elements emerging.

Table 5: Additive constant and strong performing elements for total panel, two complementary mind-sets, three complementary mind-sets, and four complementary mind-sets, respectively. Only elements showing a coefficient of +6 or higher are shown.

\begin{tabular}{|c|c|c|c|c|c|c|c|c|c|c|c|}
\hline & & \multirow[t]{2}{*}{ Tot } & \multicolumn{2}{|c|}{ Two Mind-Sets } & \multicolumn{3}{|c|}{ Three Mind-Sets } & \multicolumn{4}{|c|}{ Four Mind-Sets } \\
\hline & & & $2 \mathrm{~A}$ & $2 B$ & $3 \mathrm{C}$ & $3 \mathrm{D}$ & $3 \mathrm{E}$ & $4 \mathrm{~F}$ & $4 \mathrm{G}$ & $4 \mathrm{H}$ & $4 \mathrm{I}$ \\
\hline & & & $\stackrel{\rho}{\mathscr{8}}$ & 莺 & 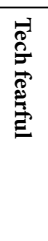 & 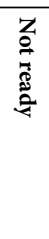 & 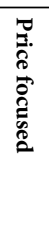 & 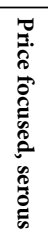 & 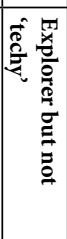 & 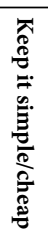 & 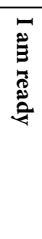 \\
\hline & Base Size & 50 & 44 & 6 & 31 & 6 & 13 & 19 & 12 & 6 & 13 \\
\hline & Additive constant) - Basic likelihood of switching w/o messaging & 20 & 21 & 18 & 23 & 18 & 16 & 29 & 13 & 18 & 16 \\
\hline A2 & Check service problems online! Our online support staff can keep you updated on all service problems. & & & & 6 & & & 10 & & & \\
\hline $\mathrm{C} 1$ & Individual plans start as low as $\$ 39.99$ a month & & & 6 & & 6 & 9 & 8 & & 6 & 9 \\
\hline $\mathrm{C} 4$ & A 2-gigabyte data plan for only $\$ 35$ a month & & & & & & 7 & 8 & & & 7 \\
\hline $\mathrm{C} 2$ & Family plans starting from $\$ 59.99$ & & 6 & & & & 6 & 7 & & & 6 \\
\hline D5 & Choose from our wide (Product 3) selection for web browsing and mobile apps & & & & & & 8 & & 8 & & 8 \\
\hline $\mathrm{D} 2$ & Don't like too many buttons... We also carry simple touch tone phones & & & 6 & & 6 & & & & 6 & \\
\hline A5 & Check your phone and voice your concerns at any of our retail stores & & & & 7 & & & & 9 & & \\
\hline D4 & Star Wars lover... Our (Product 2) phone has an awesome Star Wars theme. & & & & & & 6 & & & & 6 \\
\hline C3 & Don't want a plan... pre-paid service for only $\$ 2$ a day & & & & & & 6 & & & & 6 \\
\hline B5 & Applications can be purchased online and downloaded immediately to your phone & & & & & & & & 9 & & \\
\hline D6 & Our amazing (Product 4) phone also features a slide out keyboard & & & & & & & & 8 & & \\
\hline B1 & Most of our phones come with pre-installed cameras, games, and applications & & & & & & & & 7 & & \\
\hline B6 & Many different pricing options for applications -from monthly subscription to one-time fees & & & & & & & & 6 & & \\
\hline A4 & Stores everywhere to help you find the right phone & & & & & & & & 6 & & \\
\hline A6 & Reasonably priced extended warranties for all of our phones . & & & & & & & & 6 & & \\
\hline B4 & All compatibility questions can be easily answered online on our website & & & & & & & & & 6 & \\
\hline A1 & Hundreds of technical support staff only a phone call away & & & & & & & & 6 & & \\
\hline
\end{tabular}


When we move to three mind-sets we see seven elements emerging. When we move to four mind-sets, we also see the same seven elements emerging, and some very strong performances. The key group on which to focus is Mind-Set 4F, first because it is the largest mind-set (19 respondents), and second because it has the highest basic likelihood to switch, based on the additive constant of 29 . Table 4 is sorted by Mind-Set $4 \mathrm{~F}$ of the four mind-sets. These are the price-focused, and the serious users. The key messages are:

A2: Check service problems online! Our online support staff can keep you updated on all Service problems.

\section{C1: Individual plans start as low as \$39.99 a month}

The offering can be improved by choosing a message which also appeals to Mind-Set 4G, those who like to explore, but are not technically adept (not 'techy')

A5: Check your phone and voice your concerns at any of our retail stores

\section{Discussion and Conclusions}

Everyday life is replete with opportunities to understand the way people make decisions. The common approach is to look at the problem from the 'outside-in,' searching for regularities, and patterns. This approach characterizes a great deal of what we know about consumers. The academic literature focuses on the pattern, the generalities, the so-called 'nomothetic', coined from the Greek word Nomos, pertaining to the general, the normative.

We can trace this focus of outside-in to the development of science, where the focus is on discovering patterns, and where there was no 'mind' to report the experience, other than the mind of the researcher. This attitude of searching for patterns is important in the world of science, where the focus is on discovering patterns in a nature which has no 'communicating mind.' The reality is that searching for patterns, running experiments and measuring results, are the only ways of making sense out of nature which is mute, but lawful.

The opportunity learns about patterns of thinking and patterns of behavior are much different when we work with people who can talk. Two different measures emerge. The first is what people say they will do, and second is what people actually do. Up to now the focus of 'real science' has been on measuring what people actually do. That measure is considered the 'real' information. What people say they do is cast off as attitudes, something to measure, but not necessarily something on which to establish a science. It is precisely the patterns of what people 'say they will do' with different stimuli and in varying situations which constitutes the basis of Mind Genomics.

At the practical level, the data just shown suggests a richness of understand to be had of the world of the everyday by doing the simple experiments prescribed by Mind Genomics. The data may well enhance business performance on the one hand, as it enhances our knowledge of people and motives on the other. Examples include studies on attendance at museum by teens [10], and the recognition that entire world of new knowledge awaits the Mind Genomics researcher $[11,12]$.

\section{Acknowledgment}

The author wishes to thank Professor Martin Braun of Queens College, and Professor Sue Henderson of New Jersey City University, who were instrumental in the work at Queens College leading to these Mind Genomics studies. The studies were done by the Ms. Janna Kaminsky and the late Stephen Onufrey, in Math 110.

\section{References}

1. Ranganathan C, Seo D, Babad Y (2006) Switching behavior of mobile users: Do users' relational investments and demographics matter? European Journal of Information Systems 15: 269-276.

2. Grigoriou N, Majumdar A, Lie L (2018) Drivers of brand switching behavior in mobile telecommunications. Athens Journal of Mass Media and Communications 4: 7-28.

3. Moskowitz HR (2012) 'Mind Genomics': The experimental, inductive science of the ordinary, and its application to aspects of food and feeding. Physiology \& behavior 107: 606-613. [crossref]

4. Moskowitz HR, Gofman A, Beckley J, Ashman H (2006) Founding a new science: Mind genomics. Journal of Sensory Studies 21: 266-307.

5. Porretta S (2021) The Changed Paradigm of Consumer Science: From Focus Group to Mind Genomics. In Consumer-based New Product Development for the Food Industry Royal Society of Chemistry 21-39.

6. Porretta S, Gere A, Radványi D, Moskowitz H (2019) Mind Genomics (Conjoint Analysis): The new concept research in the analysis of consumer behaviour and choice. Trends in Food Science \& Technology 84: 29-33.

7. Lundstedt T, Seifert E, Abramo L, Thelin B, Nyström Å, et al. (1998) Experimental design and optimization. Chemometrics and Intelligent Laboratory Systems 42: 3-40.

8. Gofman A, Moskowitz H (2010a) Isomorphic permuted experimental designs and their application in conjoint analysis. Journal of Sensory Studies 25: 127-145.

9. Fraley C, Raferty AE (1998) How many clusters? Which clustering method? Answers via model-based cluster analysis. The Computer Journal 41: 578-588.

10. Gofman A, Moskowitz HR (2010b) Improving customers targeting with shor intervention testing. International Journal of Innovation Management 14: 435-448.

11. Milutinovic V, Salom J (2016) Mind Genomics: A Guide to Data-Driven Marketing Strategy. Springer.

12. Gofman A, Moskowitz HR, Mets T (2011) Marketing museums and exhibitions: What drives the interest of young people. Journal of Hospitality Marketing \& Management 20: 601-618.

\section{Citation:}

Moskowitz H (2021) Convincing Prospects to Switch Mobile Phone Providers: A Mind Genomics Cartography of an Everyday Opportunity to Optimize Messaging. Mind Genom Stud Psychol Exp Volume 1(1): 1-9. 